\title{
Necrotizing leukocytoclastic vasculitis mimicking necrotizing fasciitis: a case report
}

\author{
Mehmet Salih Soylemez ${ }^{*}$, Korhan Ozkan², Bulent Kılıc ${ }^{3}$, Samet Erinc ${ }^{2}$, \\ Irfan Esenkaya ${ }^{2}$, Bahar Ceyran ${ }^{4}$
}

\author{
${ }^{1}$ Department of Orthopaedics and Traumatology, Bingol State Hospital, Bingol, Turkey \\ ${ }^{2}$ Department of Orthopaedics and Traumatology, ${ }^{4}$ Department of Pathology, Istanbul Medeniyet University, Goztepe \\ Training and Research Hospital \\ ${ }^{3}$ Department of Health Sciences, Istanbul Gelisim University, Istanbul, Turkey
}

Received: 19 July 2016

Accepted: 11 August 2016

\section{*Correspondence:}

Dr. Mehmet Salih Soylemez,

E-mail: slhsylmz@gmail.com

Copyright: (C) the author(s), publisher and licensee Medip Academy. This is an open-access article distributed under the terms of the Creative Commons Attribution Non-Commercial License, which permits unrestricted non-commercial use, distribution, and reproduction in any medium, provided the original work is properly cited.

\begin{abstract}
There are several subtypes of necrotizing leukocytoclastic vasculitis, which are classified according to their morphological features in biopsy specimens using immunofluorescence microscopy. Necrotizing leukocytoclastic vasculitis is limited to the skin, predominantly that of the lower extremities, and usually spares the palms and soles. The most common skin manifestation is palpable purpura. Other skin manifestations include maculopapular rash, bullae, papules, nodules, ulcers and livedo reticularis. There is no specific laboratory test to determine the diagnosis. There are various diseases presenting with these nonspecific symptoms, and a rapid differential diagnosis must be conducted, because the appropriate differentiation and diagnosis markedly influence the treatment strategy and survival of patients. In this study, we report a case of necrotizing leukocytoclastic vasculitis presenting with internal organ involvement and symptoms of necrotizing fasciitis, with emphasis on the clinical differentiation.
\end{abstract}

Keywords: Leukocytoclastic vasculitis, Differential diagnosis, Necrotizing fasciitis

\section{INTRODUCTION}

Necrotizing leukocytoclastic vasculitis (NLV) (also called cutaneous leukocytoclastic vasculitis or necrotizing vasculitis) is a necrotizing inflammation of the small vessels in the dermis. The pathogenesis of NLV is generally believed to be mediated by autoimmune processes and circulating immunocomplexes deposited in the involved vessel walls. ${ }^{1}$

NLV may be associated with bacterial, viral or fungal infections, drug ingestion, inflammatory chronic systemic diseases, rheumatic diseases and haematological malignancies, including leukaemia, lymphoma, myeloma, cryoglobulinemia and myelodysplastic syndrome. ${ }^{1,2}$ However, the etiology is unknown in approximately $60 \%$ of cases. ${ }^{3}$ The clinical presentation of NLV varies and may consist of macules, pustules, vesicles, nodules, ulcerative lesions and papulonodular necrotic lesions on the extensor surfaces of the lower limbs and granulomatous lesions. ${ }^{3}$

However, necrotizing fasciitis is a rare, rapidly progressing and often mortal soft-tissue infection most commonly resulting from polymicrobial infections. The process is characterized by extensive necrosis of the subcutaneous tissue and deep fascia resulting from microvascular occlusion. ${ }^{4}$

NLV manifesting with the clinical features of necrotizing fasciitis is a rare situation and may have catastrophic results if a differential diagnosis cannot be determined at the time. In this study, we report a case of necrotizing leukocytoclastic vasculitis presenting with internal organ involvement and symptoms of necrotizing fasciitis, emphasizing the clinical differentiation. 


\section{CASE REPORT}

A 53-year-old man was admitted to the internal disease clinic of our hospital because of swelling, tenderness and hyperaemia on his left leg. The patient had a medical history of pemphigus vulgaris, which was in remission. After evaluation, the patient was transferred to the ward with a diagnosis of acute renal failure and cellulitis on his left leg to be further evaluated. In the internal disease clinic, the patient was started on antibiotics, and possible causes of the acute renal failure were investigated. However, 3 days after the initial admittance, the patient was transferred to the intensive care unit with a diagnosis of systemic inflammatory response syndrome (SIRS).

The patient was referred to our orthopaedic surgery clinic at this stage with a possible diagnosis of necrotizing fasciitis indicated by ulcerative, bleeding, wide-skin lesions starting from the extensor face of the right thigh and extending to the ankle. On physical examination, he was not well oriented and was pale and febrile $\left(39.5^{\circ} \mathrm{C}\right)$, with a pulse rate of $138 / \mathrm{min}$, a blood pressure of $75 / 45$ $\mathrm{mmHg}$ under dopamine, a respiratory rate of $35 / \mathrm{min}$ and an oxygen saturation of $59.8 \%$ on mask air. There was intercostal retractions during breathing. Blood counts showed the following: white blood cell count, 19,900 $\mathrm{mm}^{3}$; haemoglobin level, $12.4 \mathrm{~g} / \mathrm{dL}$; $\mathrm{C}$ reactive protein level, $31.5 \mathrm{mg} / \mathrm{dL}$; sodium level, $131 \mathrm{mEq} / \mathrm{L}$; creatinine level, $3.4 \mathrm{mg} / \mathrm{dL}$; and potassium level, $4.2 \mathrm{mEq} / \mathrm{L}$. The reason for the acute renal failure was still unknown, and the patient underwent dialysis.

There were wide, erythematous, ulcerative and bleeding skin lesions starting from the anterolateral aspect of the left thigh and extending to the ankle. The lesions were not continuous, and healthy skin areas were present between the lesions as in Figure 1.

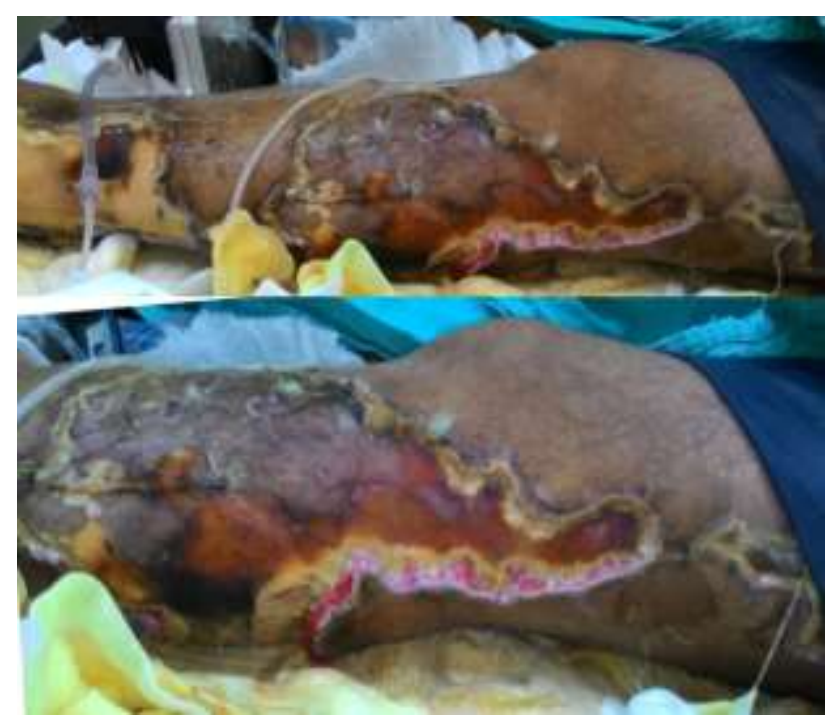

Figure 1: Clinical appearance of cutaneous lesions on the lateral aspect of the left leg 1 week after the initial admission.
There was no crepitus on the skin. The popliteal artery, dorsalis pedis artery and posterior tibial artery pulses were palpable. Conventional radiographs of the patient revealed no subcutaneous gas areas or erosion on the bones. Doppler ultrasound of the arteries demonstrated normal flow in all arteries. Magnetic resonance imaging (MRI) demonstrated suspicious oedematous involvement in subcutaneous areas as shown in Figure 2.
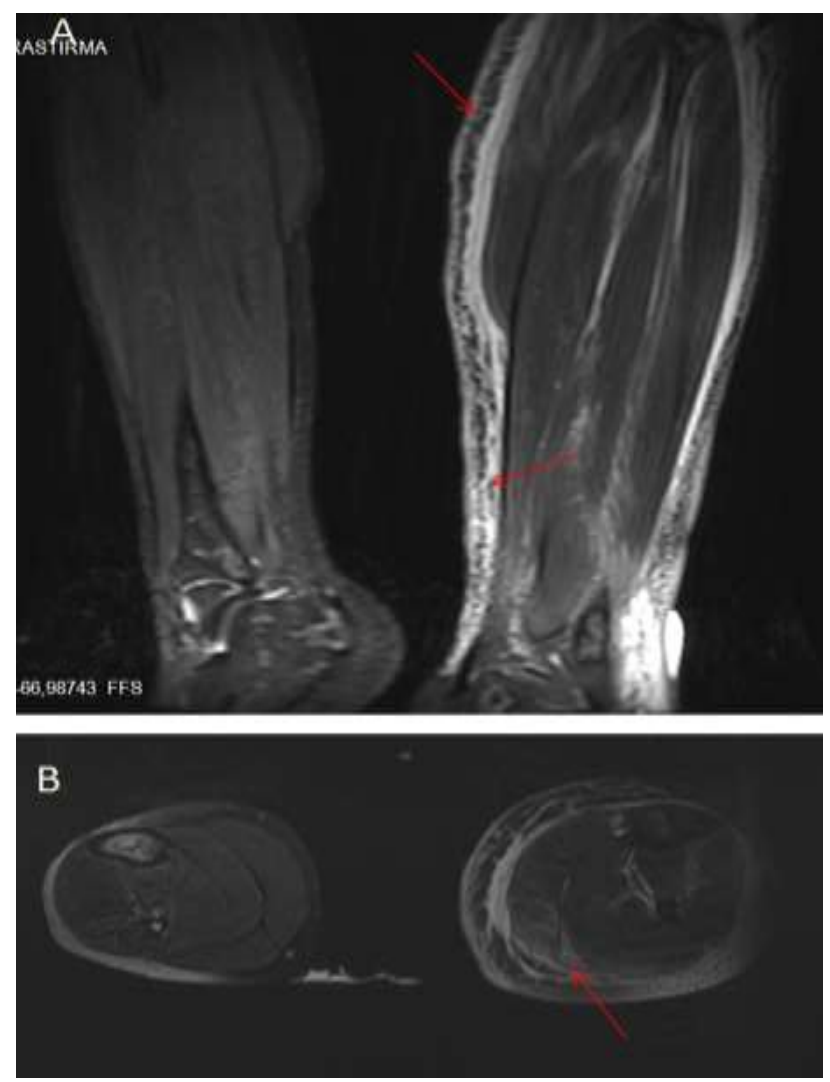

Figure 2: A) STIR coronal MRI; red arrows showing subcutaneous edema. B) T2-weighted axial MRI; the red arrow shows oedematous involvement at the musculature and interfascial plane.

Although x-ray and Doppler ultrasound images were normal, oedematous involvement on MRI and the existence of SIRS were suggestive of necrotizing fasciitis. Thus, the patient underwent immediate surgery to either exclude necrotizing fasciitis from the differential diagnosis or determine whether to perform wide debridement or amputation according to the involvement of deep tissues.

A longitudinal $5 \mathrm{~cm}$ incision was made on the lateral aspect of the left cruris. There was no abscess, subcutaneous edema or involvement in the deep fascia. There was no evidence of necrotizing fasciitis or any other infectious process. Initial Gram staining revealed no organisms present. Cultures and biopsies were taken from all layers of the skin and tissue for microbiological and histopathological investigations. All of the lesions were closed using vacuum-assisted closure with an airtight 
dressing. Intravenous wide-spectrum antibiotics were initiated as a prophylactic treatment to prevent a superinfection.

Skin biopsy revealed excess neutrophils accumulated around the vessels of the dermis resulting in vessel wall damage. A possible diagnosis of necrotizing leukocytoclastic vasculitis was proposed as in Figure 3.

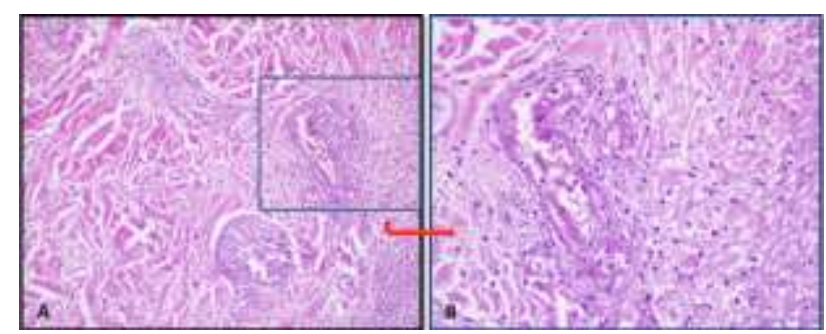

Figure 3: A) H\&E, $200 \mathrm{X}$. Microscopic image showing necrosis in the soft tissue surrounding the small vessels of the dermis. Fibrin in the walls of venules caused endothelial thickening and endothelial proliferation. Obliteration of the vessels resulting from excessive neutrophilic collection. B) H\&E $400 \mathrm{X}$ microscopic image showing neutrophilic infiltration and fibrinoid necrosis areas containing eosinophils. Neutrophils and nuclear dust in and around the walls of venules (leukocytoclasis) $(\mathrm{H} \& \mathrm{E}$; hematoxylin and eosin).

However, immunofluorescence microscopy could not be performed, and the disease subtype was not determined. The patient consulted with the dermatology clinic after surgery. Corticosteroid treatment was applied at $3 \times 500$ $\mathrm{mg} /$ day over 3 days with intervals of 5 days. Cultures taken from deep soft tissue and fascia revealed no organisms present. After the corticosteroid regimen, the lesions regressed, and routine kidney function tests were within the normal ranges. Daily dressing and debridement of the remaining lesions were performed with the help of plastic surgeons over the following days. After 45 days of follow-up, all of the lesions were remediated, and the patients' routine kidney function tests were within the normal ranges as given in Figure 4.

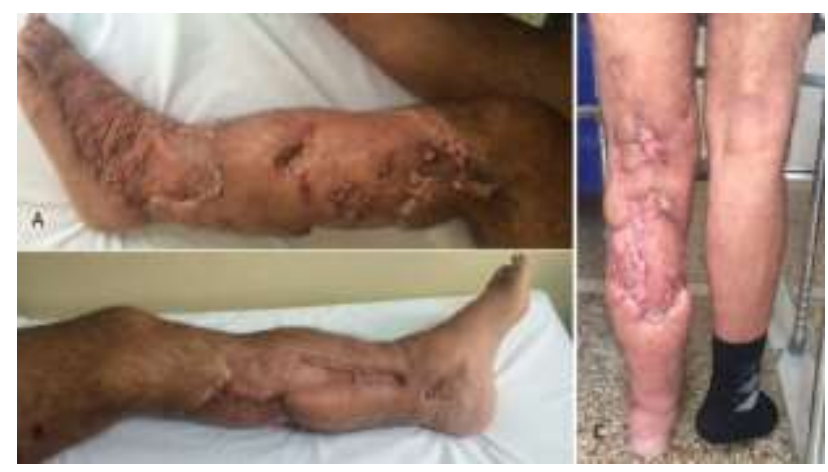

Figure 4: A) lateral, B) medial and C) posterior clinical appearance of the leg 3 months after the initial admission.

\section{DISCUSSION}

Leukocytoclastic vasculitis is a general term used to define a type of vasculitis that affects small venules (venulitis). There are several subtypes of this condition, and they are classified according to their morphological features in biopsy specimens using immunofluorescence microscopy. ${ }^{5}$ However, the definition used for this group of disorders varies from one study to another. ${ }^{1-3,6} \mathrm{We}$ prefer to use the term NLV, which we believe better defines the clinical condition of our case.

NLV is limited to the skin, predominantly the lower extremities, and usually spares the palms and soles. The most common skin manifestation is palpable purpura. Other skin manifestations include maculopapular rash, bullae, papules, nodules, ulcers and livedo reticularis. ${ }^{7}$ There are no specific laboratory tests for NLV. The diagnosis is based on the clinical picture and histopathologic features of the skin biopsy. ${ }^{8}$ Internal organ involvement is rare, and renal involvement is the most common manifestation. ${ }^{9}$

There are various diseases that present with nonspecific symptoms as seen in our case, and a rapid differential diagnosis must be determined, since the appropriate differentiation and diagnosis markedly influence the treatment strategy and survival of patients. ${ }^{7}$ The NLV subtypes Finkelstein's acute haemorrhagic edema of the skin, Henoch-Schoenlein purpura, microscopic polyarteritis nodosa or microscopic polyangiitis were considered in the differential diagnosis and were evaluated by internalists upon initial admission to the internal disease clinic. ${ }^{9,10}$ However, dramatic progression of cutaneous necrosis and worsening of the general condition of the patient prompted us to consider purpura fulminans and necrotizing fasciitis as differential diagnoses.

Purpura fulminans manifests with intravascular thrombosis and haemorrhagic infarction of the skin that is rapidly progressive and accompanied by vascular collapse and disseminated intravascular coagulation. ${ }^{11}$ Purpura fulminans was excluded from the differential diagnosis, because Doppler ultrasonography revealed normal findings, and the complete blood count (CBC) results were not congruent with disseminated intravascular coagulation.

Necrotizing fasciitis is a rare, rapidly progressive and often mortal soft-tissue infection most commonly resulting from polymicrobial infection. The process is characterized by extensive necrosis of the subcutaneous tissue and deep fascia resulting from microvascular occlusion. Causative infectious agents tend to be both aerobic and anaerobic organisms. ${ }^{4,12}$ The infection typically begins as a localized abscess, particularly among at-risk groups, such as intravenous drug users, patients with diabetes mellitus, alcoholics, those undergoing post-abdominal surgery, obese individuals or 
patients with either perineal infections or peripheral vascular disease. ${ }^{13}$ The entity is considered a medical emergency, with mortality rates reported as high as 70 $80 \%$, and death is most commonly caused by disseminated intravascular coagulopathy, sepsis or respiratory, renal or multisystem organ failure. ${ }^{4,14}$ Subcutaneous gas is a pathognomonic radiological finding in patients without a history of trauma, but it may not be present initially, and diagnoses may be missed in patients presenting with mild symptoms such as pain, edema and tachycardia. MRI findings and Gram staining of soft tissue are usually diagnostic in suspected cases. ${ }^{14}$

Limited time was the most challenging situation in this case. Because the patient was referred to our clinic after 3 days of symptoms, the medical history was congruent with necrotizing fasciitis, and the etiology of acute renal failure remained unknown. SIRS can be caused by necrotizing fasciitis, and in such cases, immediate amputation can save the patient. However, an unnecessary amputation performed without sufficient evidence may have catastrophic results for both the patient and clinician. Thus, a rapid radiographic assessment (X-ray, MRI, Doppler ultrasound) was performed. Although X-rays and Doppler ultrasonography revealed normal findings, MRI demonstrated suspicious oedematous involvement in the subcutaneous area, which is suggestive of necrotizing fasciitis. ${ }^{12}$ After only 1 hour, we performed diagnostic surgery.

After diagnostic surgery, the diagnosis of necrotizing fasciitis was eliminated, because all possible diagnostic measures were performed, and the involvement of deep tissue or fascia was not detected in any way.

The etiology remains unknown in our case. Our patient had a history of pemphigus vulgaris. Some of the medical agents used for the treatment of pemphigus vulgaris (sulfasalazine, methotrexate) have been reported to cause leukocytoclastic vasculitis. ${ }^{15}$ However, this disorder was in remission, and the patient had not taken any medications for the last 2 months. However, NLV after rheumatic diseases has been reported, and pemphigus vulgaris may have triggered the NLV, although there is no evidence in this case. ${ }^{1}$

The treatment of NLV remains controversial. ${ }^{16}$ The diseases that occur after medical agent use tend to recover after cessation of the suspected agent. Colchicine and dapsone may be used in patients with limited palpable purpura. ${ }^{8}$ However, after initiation of the ulcerative lesions, these agents are ineffective. ${ }^{7}$ Corticosteroids are widely used in inflammatory disorders to control the local inflammatory response that causes ischemia. Additionally, they have been used for the treatment of NLV. However, contrary to our case, they have been reported to be ineffective or to have a lower efficiency than expected. ${ }^{17}$ In our case, after initiation of the intravenous corticosteroid regimen, a dramatic regression of the lesions and remission of the patient were observed.
Only one case with similar cutaneous features was reported by Brown et al. ${ }^{7}$ That case, who presented with similar cutaneous features on the hand and cruris, was diagnosed with vasculitis due to naproxen usage 1 week after the initial admission. However, the patient exhibited sustained thromboemboli and underwent amputation of her finger and cruris despite treatments with all suggested medical agents, including corticosteroids, colchicine, dapson cyclophosphamide, sildenafil and heparin.

\section{CONCLUSION}

The clinical features of NLV rarely progress aggressively; in such cases, the diagnoses may be delayed, resulting in unforeseen morbidities or even mortality. As far as we know, this is the second case of leukocytoclastic vasculitis manifesting with large areas of cutaneous necrosis that mimic necrotizing fasciitis. A fast and rigorous radiological and clinical evaluation must be performed to obtain an early differential diagnosis, which will affect the treatment strategy. The early initiation of required treatment may prevent mortalities and morbidities, as seen in our case.

Funding: No funding sources Conflict of interest: None declared

Ethical approval: Not required

\section{REFERENCES}

1. Koutkia P, Mylonakis E, Rounds S, Erickson A. Leucocytoclastic vasculitis: an update for the clinician. Scand J Rheumatol. 2001;30(6):315-22.

2. Das M, Chhabra R, Hinton SW. Cutaneous leukocytoclastic vasculitis and myelodysplastic syndrome with little or no evidence of associated autoimmune disorders - a case report and a brief review of the literature. Am J Med Sci. 2008;336(4):368-71.

3. Tsampau D, Buggiani G, Hercogova J, Lotti T. Cutaneous necrotizing vasculitis: a rational therapeutic approach. Dermatol Ther. 2012;25(4):335-9.

4. Chaudhry AA, Baker KS, Gould ES, Gupta R. Necrotizing fasciitis and its mimics: what radiologists need to know. AJR. Am. J. Roentgenol. 2015;204:128-39.

5. Smoller BR, McNutt NS, Contreras F. The natural history of vasculitis. What the histology tells us about pathogenesis. Arch Dermatol. 1990;126(1):84-9.

6. Dinato SL, Dinato MC, Dinato MM, Ribeiro MC, Yamashiro CY, Romiti N. Necrotizing vasculitis as a manifestation of hypersensitivity to propylthiouracil]. Arq Bras Endocrinol Metabol. 2009;53(3):374-7.

7. Brown K, Martin J, Zito S. Severe leukocytoclastic vasculitis secondary to the use of a naproxen and requiring amputation: a case report. J Med Case Rep. 2010;4:204. 
8. Pongruangporn M, Ritchie DJ, Lu D, Marschall J. Vancomycin-associated leukocytoclastic vasculitis. Case Rep Infect Dis. 2011;2011:356-70.

9. Tancrede-Bohin E, Ochonisky S, Vignon-Pennamen M-D, Flageul B, Morel P, Rybojad M. SchonleinHenoch purpura in adult patients: 438 predictive factors for IgA glomerulonephritis in a retrospective study of 57 cases. Arch Dermatol. 1997;133(4):43842.

10. Irvine AD, Bruce IN, Walsh MY, Bingham EA. Microscopic polyangiitis: Delineation of a cutaneous-limited variant associated with antimyeloperoxidase autoantibody. Arch Dermatol. 1997;133(4):474-7.

11. Edlich RF, Cross CL, Dahlstrom JJ, Long WB: Modern concepts of the diagnosis and treatment of purpura fulminans. J Env Pathol Toxicol Oncol. 2008;27(3):191-6.

12. Schmid MR, Kossmann T, Duewell S. Differentiation of necrotizing fasciitis and cellulitis using MR imaging. AJR. Am. J. Roentgenol. 1998;170:615-20.

13. Stephen R, Burnett J, Pavlides N. Musculosceletal Infections and Microbiology in AAOS. In:
Lieberman JR, eds. Comperehensive Orthopaedic Review. Illinois, USA. 2009: 98-100.

14. Wong CH, Chang HC, Pasupathy S, Khin LW, Tan JL, Low CO. Necrotizing fasciitis: clinical presentation, microbiology, and determinants of mortality. J Bone Joint Surg Am. 2003;85:1454-60.

15. Ten Holder SM, Joy MS, Falk RJ. Cutaneous and systemic manifestations of drug-induced vasculitis. Ann Pharmacother. 2002;36(1):130-47.

16. Machaczka $M$, Klimkowska $M$, Hägglund $H$. Unexpected cure from cutaneous leukocytoclastic vasculitis in a patient treated with $\mathrm{N}$ butyldeoxynojirimycin (miglustat) for Gaucher disease. Adv Med Sci. 2012;1;57(1):169-73.

17. Daniel CL. Leukocytoclastic vasculitis and HenochSchonle in Purpura in Arthritis \& Allied Conditions. Lippincott Williams \& Wilkins. 15th edition. vol. 86. Philadelphia, USA. 2005: 1793-1797.

Cite this article as: Soylemez MS, Ozkan K, Kılıc B, Erinc S, Esenkaya I, Ceyran B. Necrotizing leukocytoclastic vasculitis mimicking necrotizing fasciitis: a case report. Int J Res Orthop 2016;2:194-8. 